\title{
Investigation of a CCD modulation transfer function using the speckle method at different laser wavelengths and sub-windowing options
}

\author{
A. Nasibov, A. Kholmatov, H. Nasibov^, and F. Hacizade
}

The Scientific and Technical Research Council of Turkey, Center of Research for Advanced Technologies of Informatics and Information Security (TÜBITAK-BíLGEM), Gebze, Turkey

Received: 16 November 2010 / Accepted: 10 December 2010

\begin{abstract}
We have experimentally investigated the MTF of a scientific grade CCD, especially considering its behavior induced by different laser wavelengths (from $470 \mathrm{~nm}$ to $633 \mathrm{~nm}$ ) and varying number of total charge transfers. The MTF measurements were performed using laser speckles and utilizing the single-slit method. The MTF of the sensor was examined from zero to the Nyquist frequency. The results obtained at different wavelengths were compared. It was shown that the spectral behavior of the MTF is negligibly small; moreover the small variation is observed at the high frequency region. It was also observed that the variation of the total number of charge transfers, adjusted by size and location of the region of interest for readout pattern, is within the intrinsic error of the single-slit aperture laser speckle method.
\end{abstract}

Keywords: Scientific grade CCD, MTF; modulation transfer function; laser speckle; spatial-frequency response

\section{Introduction}

The role of the optical imaging metrology has been increasing in many fields of applied science and industry, especially where invasive methods fail, such as moving objects, mediums down to the micron size scale, hot surfaces, etc. However, the precision of measurements obtained by optical systems highly depends on the quality of the recorded images. Many metrics have been suggested for the image quality assessment [1]. Unfortunately, all of these metrics are scene and task dependent. Moreover, a general ideal metric for an imaging system's performance, that is bounded by detector and imaging optics limit, does not exist. In digital imaging systems, the performance of the imaging sensors significantly affects the quality of the output image. Among the variety of the spectrophotometrical and electronic parameters of the sensor, the signal to noise ratio and the modulation transfer function (MTF) are the essential characteristics influencing the quality of the output image. The MTF is a classic measure characterizing the spatial-frequency response of the array imaging systems [2], where the sensor's MTF limits the spatialfrequency content of the output image. In other words, the MTF gives quantitative information about imaging performance of an image-forming system, i.e. it quantifies its ability to resolve small features in an object with a particular size [3]. In general, images with the higher

^ Correspondence: humbat.nasibov@uekae.tubitak.gov.tr
MTFs and less noise are judged as having better image quality [1]. Therefore, assessment of the MTF plays a pronounced role in the design of the digital vision systems for the optical imaging metrology.

The sensor MTF is a single plot that quantifies the image contrast as a function of the spatial frequency. Commonly, the MTF is determined by the evaluation of the array response to the continuous input illumination with the intensity distribution of $I=0.5[1+\cos (s)]$, where $s$ is a general spatial parameter [4]:

$$
M T F=\frac{P_{\max }-P_{\min }}{P_{\max }+P_{\min }} .
$$

The $P_{\max }$ and $P_{\min }$ are the values of neighboring maximum and minimum of two specific pixels in the output image.

In practice, the MTF of the imaging sensor is assessed experimentally, generally by the system response to target patterns with predetermined spatial-frequency contents. Depending on the utilized input patterns, experimental methods can be divided into two groups: the deterministic and the stochastic.

Various targets with a known spatialfrequency content, such as bar target, sinusoidal patterns, interferometric fringes, canted self-imaging targets and many others are used in the deterministic methods [5-8], while in stochastic methods, also known as random target methods, patterns with randomly distributed spatial frequencies are used. The idea of using targets with random distribution of 
the spatial content firstly was suggested in [9], where it was shown that the spatial frequency distribution of the photographic emulsion silver grains on the uniformly exposed chart pattern is ergodic, and its autocorrelation function can be described analytically. This method has become widespread and is widely used along with a test input pattern generated by the laser speckle illumination [10-17]. The laser speckle method has several main advantages compared to other existing methods: (a) it does not require any imaging optics, (b) the alignment of the optical system is not critical as with the bar chart test inputs, (c) it allows characterizing entire area of the sensor.

Besides its geometrical parameters (pixel size, shape and pixel pitch), the sensor MTF also depends on many physical and electronic parameters, as well as many other underlying working principles of a particular sensor [18]. In the case of the CCD sensor, there are two major components contributing to the overall MTF, namely the diffusion and the charge transfer MTFs. In this work, we have investigated the impact of these dependencies on to the total MTF of a scientific grade CCD sensor using single-slit laser speckle method.

The paper is organized as follows: in Section 2 we briefly describe the components of the MTF essential for charge-coupled device type sensors and give the mathematical background of the single-slit laser speckle method. Section 3 outlines our experimental setup and measurement procedures. Section 4 presents experimentally obtained results of the MTF measurements at different laser wavelengths (i.e. spectral dependence of the MTF), and at various sub-windowed readout options (i.e. dependence of the CCD MTF on the number of charge transfers). Section 5 summarizes contributions of the current work and gives prospects for the future research.

\section{Theoretical background}

The MTF of CCD can be expressed as a product of two components: $\mathrm{MTF}_{\text {sensor }}=\mathrm{MTF}_{\text {integral }} \times$ $\mathrm{MTF}_{\text {electronics }}[18]$. The $\mathrm{MTF}_{\text {integral }}$ component (sometimes also denoted as $\mathrm{MTF}_{\text {geometrical }}$ ) is responsible for a fixed modulation loss that is specific to the geometry of the pixel. The geometrical MTF only depends on the pixel pitch and size, and is common to all types of pixelbased imaging sensors. The $\mathrm{MTF}_{\text {integral sets a limit to the }}$ overall spatial-frequency response of the array detector. In practice, the real sensor's MTF is always less than the $\mathrm{MTF}_{\text {integral, }}$ which is due to the modulation losses introduced by the electronics subsystem of a camera system.

Discrete image registration may be viewed as a selective weighting of an object's spatial frequency spectrum by a CCD sensor [3]. The CCD's optical transfer function (OTF) is the Fourier transform of its intensity point-spread function, which is the two dimensional spatial impulse response. Accordingly, the OTS is the complex function [19]. The modulus of the OTF is the CCD's modulation transfer function. The two-dimensional MTF of the CCD sensor with square shape pixels is given by [20]:

$$
\operatorname{MTF}(\xi)=\operatorname{sinc}^{2}\left(\frac{\pi \xi a}{2 \xi_{N y} x}\right)
$$

where, $\operatorname{sinc}(\theta)=\sin (\theta) / \theta$ is the sinc function, $a$ is pixel size, $\xi$ is the spatial frequency that corresponds to horizontal and vertical directions, $\xi_{N y}$ is the Nyquist frequency of the CCD, $x$ is the pixel pitch.

The monochromatic laser speckle pattern is widely used in stochastic MTF measurement methods, where a target with random distributed intensities from dark to the "white" patterns with a known spatial content is recorded by the imaging sensor $[13,21]$. The output power spectral density (PSD) of the speckle pattern recorded by a CCD sensor is given by [16]:

$$
\begin{aligned}
P S D_{\text {output,experimental }}(\xi, \eta) & =[\operatorname{MTF}(\xi, \eta)]^{2} \\
\times & P S D_{\text {input, theoretical }}(\xi, \eta) .
\end{aligned}
$$

The output $\operatorname{PSD} \operatorname{PSD}_{\text {output, experimental }}(\xi, \eta)$ is estimated using the image captured by the CCD under the investigation. Precisely, the output PSD corresponds to the ensemble averaged one-dimensional Fourier transforms of the horizontal rows in the image [22]:

$$
\begin{aligned}
P S D_{\text {output, experimental }}(\xi)= & \frac{1}{M} \sum_{i=0}^{M-1} \mid \\
& \times \Phi\{\operatorname{image}(0: N-1, i)\} \mid
\end{aligned}
$$

where $M$ and $N$ correspond to the number of pixels in vertical and horizontal dimensions. The $\operatorname{PSD}_{\text {output,experimental }}(\eta)$ is estimated in the same way, where the recorded image is transposed.

The input PSD of the speckle irradiance in the CCD sensor is proportional to the autocorrelation of the transmission function of the aperture and a delta function of the zero frequency [23]. The transmission function of the single-slit rectangular aperture is given by:

$$
P(x, y)=\operatorname{rect}\left(x / L_{1}\right) \operatorname{rect}\left(y / L_{2}\right) \text {. }
$$

Then the input PSD of the speckle irradiance on the CCD sensor can be calculated, as [24]:

$$
\begin{aligned}
& P S D_{\text {input, theoretical }}(\xi, \eta)=\langle I\rangle^{2} \\
& \qquad\left[\delta(\xi, \eta)+\frac{(\lambda z)^{2}}{L_{1} L_{2}} \Lambda\left(\frac{\lambda z}{L_{1}} \xi\right) \Lambda\left(\frac{\lambda z}{L_{2}} \eta\right)\right], \quad \text { (6) } \\
& \text { where, } \Lambda(x)=\left\{\begin{array}{l}
1-|x|, \text { for }|x| \leqslant 1 \\
0, \text { elsewhere }, \text { and }\langle I\rangle \text { is the aver- }
\end{array}\right.
\end{aligned}
$$

age speckle irradiance.

For the CCD with the square shape pixels of the side length equal to $a$, a generally accepted resolution corresponding to the Nyquist spatial frequency is equal to a spacing between two pixels. Assuming that the pixel fill factor is $100 \%$ and the center-to-center spacing $(\Delta x)$ is 
equal to $a$ in both directions, then the Nyquist spatial frequency of the CCD is determined as [25]:

$$
\xi_{\text {Nyquist }}=1 / 2 \Delta x, \text { and } \quad \eta_{\text {Nyquist }}=1 / 2 \Delta y .
$$

The spatial frequencies of any particular feature in the power spectrum scales as follows [6]:

$$
\xi=x / \lambda z, \text { and } \eta=y / \lambda z,
$$

where $x$ and $y$ are the corresponding aperture dimensions, $\lambda$ is the laser wavelength, $z$ is the distance between aperture and CCD sensor. For the given sizes $L_{1}$ and $L_{2}$ of the rectangular single-slit aperture, the cutoff frequency of the power spectrum can be determined from equation (8) as:

$$
\xi_{\text {cutoff }}=L_{1} / \lambda z, \text { and } \quad \eta_{\text {cutoff }}=L_{2} / \lambda z .
$$

\section{Experimental setup}

We have used a single-slit aperture modulated laser speckle method to carry out our measurements. Laser speckle patterns with desired spatial content were obtained using the experimental setup shown in Figure 1. The radiation of the laser is directed to the input port of the integrating sphere (IS) with a diameter of $154 \mathrm{~mm}$. The input and output ports of the IS were situated perpendicularly, which guaranties that the generated speckle pattern doesn't contain specularly reflected component. The laser beam, after multiple scatterings inside the IS (coated with the barium sulfate paint), is formed at the output port of the IS. The speckle pattern has uniform intensity. On the other hand, the polarization of the laser beam at the exit port of IS becomes completely depolarized and has a uniform distribution of the phase in the $0-2 \pi$ range. The linear polarizer $\mathrm{P}$ is placed behind the aperture, so the speckle irradiance incident onto the CCD imaging sensor is plain polarized. In our experiments a monochrome CCD camera with $1360 \times 1024$ active square pixels was investigated. The center-to center spacing of pixels is $4.65 \mu \mathrm{m}$. A square single-slit aperture, with side dimensions of $2 \mathrm{~mm}$ was placed immediately at the output port of the IS. The dimensions of the slit were measured using microscope system with a telecentric objective, calibrated against a reticle. The overall uncertainty of these measurements does not exceed $\pm 0.01 \mathrm{~mm}$. The camera was mounted on the linear translation stage, which can be moved to a desired distance from the aperture using the homemade software. The spectral dependence of the total MTF was assessed using the 5 colors HeNe laser operating at $543 \mathrm{~nm}, 594 \mathrm{~nm}, 604 \mathrm{~nm}, 612 \mathrm{~nm}$, and $633 \mathrm{~nm}$ wavelengths, and two diode lasers with $470 \mathrm{~nm}$ and $535 \mathrm{~nm}$ wavelengths. The image recording software was setting the camera exposure time such that every recorded speckle pattern image had its maximum grayscale values slightly less than the saturation level. This allowed us avoiding the influence of laser's output power variation to the experimental results and obtaining uncorrelated speckle images with a maximum signal to noise ratio.

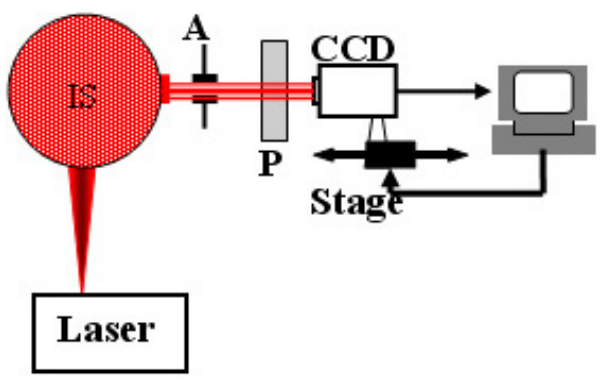

Fig. 1. Experimental setup (A - is a single slit square shaped aperture, $\mathrm{P}$ - is a plane polarizer, IS - is an integrating sphere).

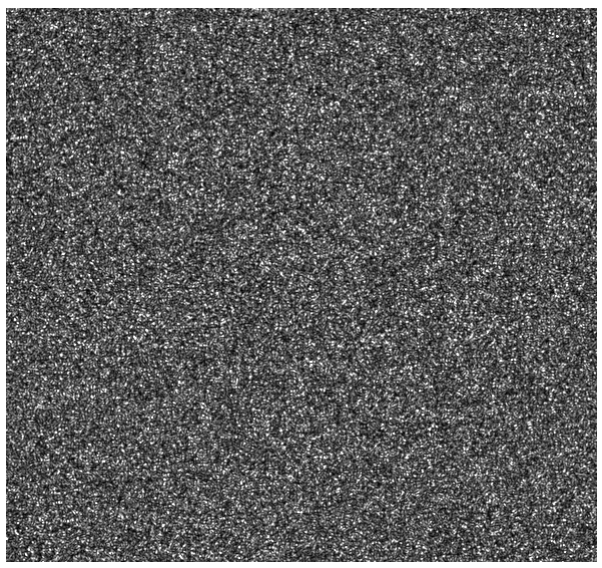

Fig. 2. A typical speckle pattern.

The influence of the readout process to the MTF was investigated by comparative study of the MTFs calculated at various sub-windowing options. For this purpose once the CCD was positioned to the proper distance corresponding to the used laser wavelength, the home made software adjusted the ROI (region of interest) parameters of the sensor and captured its corresponding speckle pattern for further calculations. To avoid aliasing effects, a sensor was placed at such a distance $z$ from the aperture that the cutoff frequency on the sensor's surface plane corresponded to the Nyquist spatial frequency of the sensor. In [19] it was shown that the distance between the aperture and the sensor plays a crucial role in the accuracy of the single-slit aperture laser speckle method, and it was reported that even a variation of $1 \mathrm{~mm}$ in this distance can cause a root-mean-square error higher than $10 \%$ in the overall MTF assessments. Moreover, a protective glass window in front of the sensor hinders performing precise measurements of the distance $z$. In experiments we have applied the procedures proposed in [12], and analyzed the behavior of the MTF curves at the high-spatial-frequency region at various distances close to $z$. By this way we have found out that the proper distance for our particular CCD is about $1.2 \mathrm{~mm}$ shorter than $z$, which is due to the thickness of the protective glass window and a gap between sensor plane and this window (according to the manufacturer datasheet the thickness of the window is $0.75 \mathrm{~mm}$ and the refractive index is 1.5). Figure 2 shows an example of the speckle pattern obtained at $535 \mathrm{~nm}$ laser wavelength. 
Table 1. Laser wavelengths and their corresponding aperture separation distances, used in the experiments.

\begin{tabular}{cc}
\hline Wavelength $(\mathrm{nm})$ & Distance $z(\mathrm{~mm})$ \\
\hline 470 & 39.5 \\
535 & 34.7 \\
543 & 34.3 \\
594 & 31.3 \\
604 & 30.8 \\
612 & 30.4 \\
633 & 29.4 \\
\hline
\end{tabular}

\section{Results and discussions}

In this section we present our experimental results and compare them to the theoretically expected results. The Nyquist frequencies of the investigated sensor in both vertical and horizontal directions were calculated as 107.53 cycles $/ \mathrm{mm}$ using equation (7). In order to avoid aliasing effects, which may be introduced by the single slit aperture laser speckle method, the CCD was placed at a distance $z$ apart from the aperture, such that the $\xi_{\text {cutoff }}$ frequency was equal to the corresponding Nyquist spatial frequency [12]. According to equation (9), the separation distance $\mathrm{z}$ depends on the laser wavelength. Thus, we have calculated relevant separation distances $z$ for each corresponding wavelength and summarized these in Table 1.

Due to the geometry of the aperture, the calculations of the MTF in horizontal and vertical directions were performed separately. The calculation of the output PSDs in each direction were performed using equation (4), where one-dimensional Fourier transforms were applied to the horizontal rows and vertical columns, separately. The vertical PSDs were similarly calculated, where the speckle pattern images were transposed prior to calculations. In order to eliminate the errors caused by the discrepancy between the sides of the square slit (less then $0.01 \mathrm{~mm}$ ), the speckle pattern images were recorded at two different positions of the aperture (normal and rotated by 90 degrees around the optical axis) and the PSDs were calculated by averaging results obtained for both positions. In the calculations more than 100 frames of the dark signal subtracted images were used to achieve statistically significant results. Figure 3 shows typical normalized PSDs of the laser speckle patterns obtained at the $535 \mathrm{~nm}$ wavelength, where the blue dotted line and the red scattered curve represent the theoretically calculated and experimentally obtained PSDs, respectively. Additionally, a third order polynomial, depicted as a black solid line in Figure 3, was fitted to the experimental PSD data. The PSDs in the horizontal and vertical directions are ranged from 0 to 107.53 cycles $/ \mathrm{mm}$ and are in good agreement with each other, which is as expected for the pixels with a square shape. Finally, the MTF of the CCD sensor was evaluated using equation (3):

$$
\operatorname{MTF}(\xi, \eta)=\sqrt{\frac{P S D_{\text {output,experimental }}(\xi, \eta)}{P S D_{\text {input }, \text { theoretical }}(\xi, \eta)}} .
$$

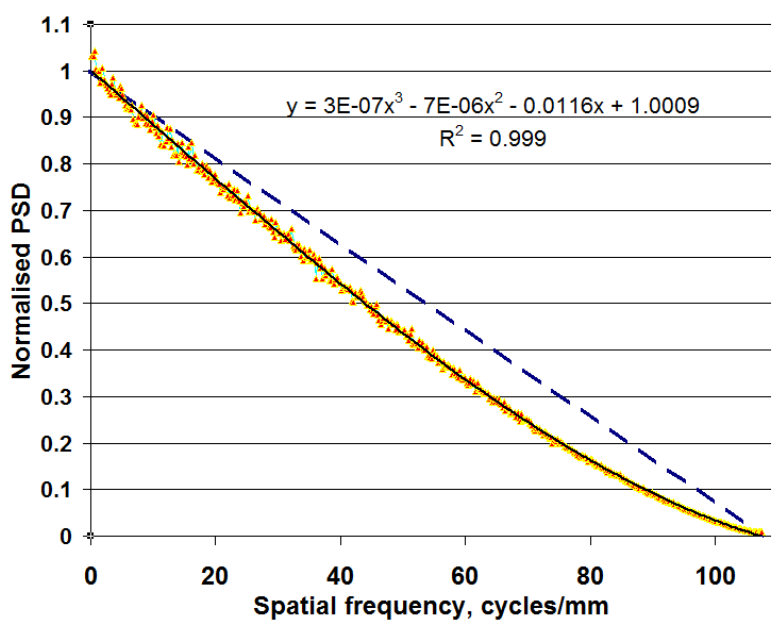

Fig. 3. Normalized PSDs: blue dashed line depicts theoretically calculated input PSD (using Eq. (6)), the scattered data is experimentally obtained at $535 \mathrm{~nm}$ wavelength, and black solid curve depicts the third order fit to the experimental data.

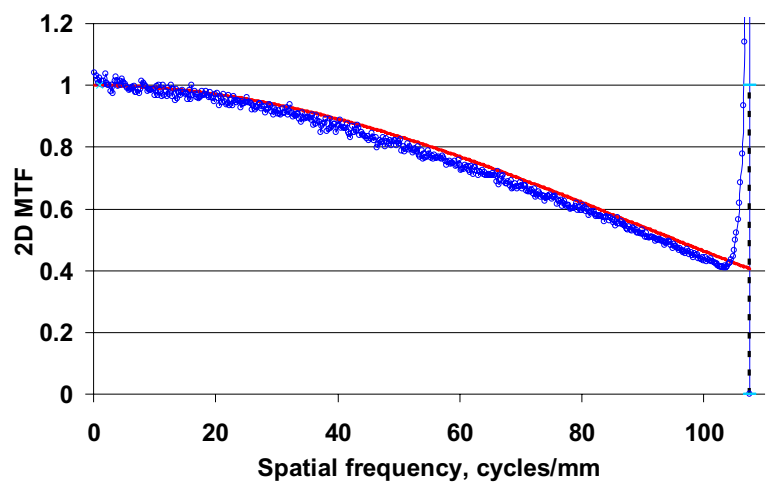

Fig. 4. Two-dimensional MTF: red solid curve depicts theoretically calculated MTF (using Eq. (2)) and the blue scattered data is experimentally obtained at $535 \mathrm{~nm}$ wavelength.

Figure 4 shows the two-dimensional MTF of the investigated sensor obtained at $532 \mathrm{~nm}$ wavelength, where the red solid curve indicates the theoretically calculated MTF (using Eq. (2)) and the black scattered curve is the experimental data. As it can be seen from Figure 4, experimentally obtained MTF curves lie slightly below their corresponding theoretically calculated curves, i.e. they suffer an additional modulation loss. Figure 5 shows the deviation of the experimentally obtained two-dimensional MTF from its theoretically calculated counterpart, where the degradation factor of the experimentally measured MTF is increasing according to the spatial frequency. In this work we aimed to investigate the behavior of the MTF degradation factor which could be influenced by different wavelengths and readout options.

Generally, equation (2) evaluates to the maximum value for the MTF corresponding to the particular spatial frequency $\xi$, which is defined by the geometry of the pixels. Where as, any modulation loss due to the fill factor, electronics subsystems and electronic image filtering and 


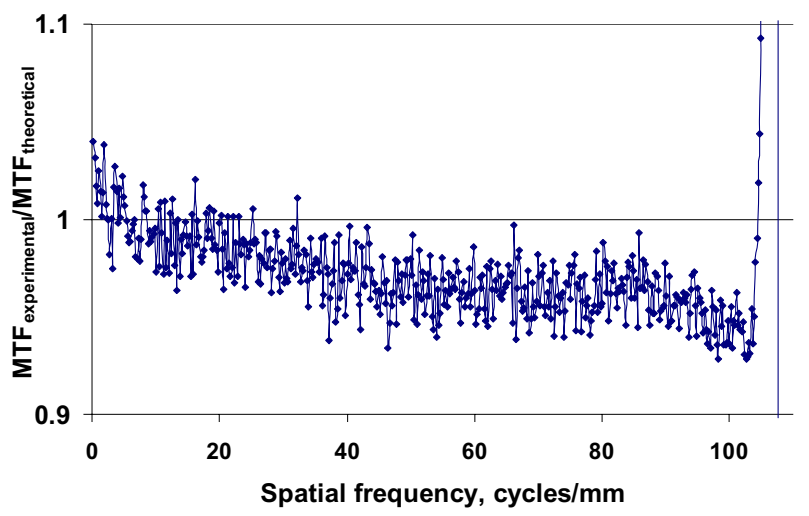

Fig. 5. Ratio of the experimentally obtained two-dimensional MTF to the theoretically calculated MTF.

construction are not taken into consideration in this equation. These types of modulation losses strongly depend on a particular type of sensor and on various physical and electronic factors. For the CCD sensors, the modulation losses due to the $\mathrm{MTF}_{\text {electronics }}$ component can be formulated as [18],

$$
M T F_{\text {electronics }}=M T F_{\text {diffusion }} M T F_{C T E},
$$

where the diffusion component $\left(\mathrm{MTF}_{\text {diffusion }}\right)$ depends on the depth of the pixel and the wavelength of the incident light. A modulation loss occurs due to the diffusion of the photon-generated carriers from one well to adjacent wells, thus causing blurs in the image. The second component, namely the charge transfer efficiency (MTF $C T E)$, accounts for the modulation loss due to the incomplete charge transfers. $\mathrm{MTF}_{C T E}$ decreases with an increasing number of charge transfers from a sensor to the output node [18].

In order to assess the variation of the overall MTF due to the $\mathrm{MTF}_{\text {diffusion }}$ component, we have measured and compared the sensor MTF at different wavelengths. It is known that, the absorption depth of photons inside a pixel well depends on the light wavelength, where the larger wavelengths generate charges towards the bottom of the pixel wells [18]. It is expected that these charges, which are being accumulated at the bottom, will diffuse from one well to another and bring extra blur to the output image.

The results of the MTF, which were calculated for wavelengths tabulated in Table 1, were compared by analyzing the second order polynomial fits to the corresponding experimental data. However to the contrary of some expectations, we have observed, that the MTF curves corresponding to the investigated laser wavelengths are very close to each other indeed. For instance, Figure 6 depicts the one-dimensional MTF curves of the CCD at the two extreme wavelengths: $470 \mathrm{~nm}$ and $628 \mathrm{~nm}$, where the largest difference between two curves is expected. The root mean square (RMS) error between MTF curves at $470 \mathrm{~nm}$ and $628 \mathrm{~nm}$ were calculated as 0.0082 . Such a low error indicates that the dependency of the MTF to the laser wavelength is negligibly small for a scientific grade CCD camera. We skip presenting RMS errors between MTF

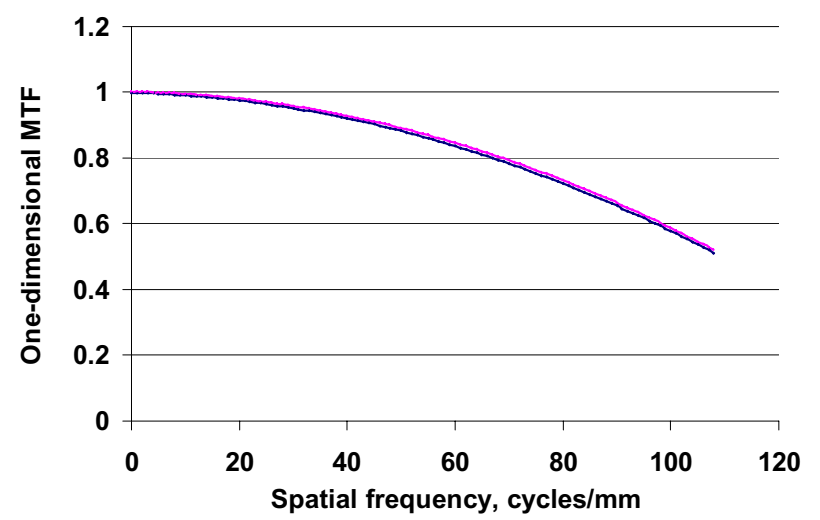

Fig. 6. Second order fits to the one-dimensional MTFs obtained at $470 \mathrm{~nm}$ (blue curve) and at $628 \mathrm{~nm}$ (red curve).

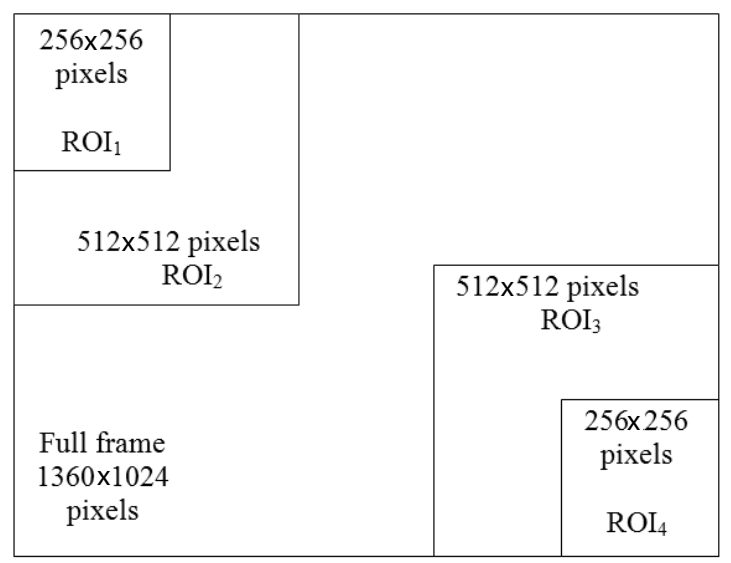

Fig. 7. Schematic representation of the ROIs used in our experiments.

curves calculated for the other wavelengths as these are even smaller than the presented one. Similar results (very low spectral dependence of the MTF of a scientific grade CCD camera) were obtained in [26].

In recent years CCD manufacturers have started supplying their devices with a flexible CCD readout pattern, which can be manipulated by the adjustable region of interest (ROI also referred as sub-windowing) and pixel binning options [18]. ROI allows selecting a specific portion of the image (commonly a rectangle) by reading charges only from corresponding pixels. By varying the area of the ROI in pixels we are able to change the total number of charge transfers, i.e. able to manipulate with the $\mathrm{MTF}_{C T E}$. Figure 7 shows the schematic representation of the ROIs used in our experiments. For this part of the work, all MTF measurements were carried out at $532 \mathrm{~nm}$ laser wavelength. Once the CCD was positioned at an appropriate distance from the aperture, the laser speckle patterns were captured at four different ROI configurations. Then, obtained results were compared to those obtained for the full frame mode. The RMS between MTFs of the $256 \times 256$ sub-window and full frame patterns was calculated as 0.0035 . Comparative analysis performed using more than 100 uncorrelated speckle patterns captured at 
various ROI configurations (as depicted in Fig. 6) and corresponding full frame patterns has shown that the variation of MTF due to the total number of charge transfers is less than the intrinsic systematic error of the single slit laser speckle method, thus can be considered as negligible.

\section{Conclusion}

The MTF is a fundamental metric characterizing the optical transfer performance of an imaging system and an efficient tool for analysing the image quality. For an ideal imaging array, the MTF is defined only by pixel size and pitch. However, for real imaging sensors it is influenced by many physical processes and electronic filtering. In this work, we investigated the impact of the incident light wavelength and total number of charge transfers to the MTF of a scientific grade CCD camera. Although the spectral sensitivity of the investigated camera is covering the visible region of the spectrum, the spectral dependence of the MTF was investigated at various laser wavelengths ranging from $470 \mathrm{~nm}$ to $628 \mathrm{~nm}$. The root mean square error between MTF curves obtained at extreme wavelengths (470 $\mathrm{nm}$ and $628 \mathrm{~nm}$ ) did not exceed 0.0082 , which shows that there is a negligible variation of the total CCD MTF due to the wavelength changing. Also, it was observed that the variation of the MTF due to the variation of total number of charge transfers did not exceed 0.0035 , which is within the intrinsic error of the applied measurement method and is negligible.

\section{References}

1. G.C. Holst, Imaging system performance based upon $F \lambda / d "$ ", Opt. Eng. 46, 103204 (2007)

2. S.K. Park, R. Schowengerdt, M. Kaczynski, Modulationtransfer-function analysis for sampled image systems, Appl. Opt. 23, 2572-2582 (1984)

3. A.D. Ducharme, S.P. Temple, Improved aperture for modulation transfer function measurement of detector arrays beyond the Nyquist frequency, Opt. Eng. 47, 093601 (2008)

4. J.C. Feltz, M.A. Karim, Modulation transfer function of charge-coupled devices, Appl. Opt. 29, 717-722 (1990)

5. D.N. Sitter Jr., J.S. Goddard, R.K. Ferrell, Method for the measurement of the modulation transfer function of sampled imaging systems from bar-target patterns, Appl. Opt. 34, 746-751 (1995)

6. N. Guérineau, J. Primot, M. Tauvy, M. Caes, Modulation transfer function measurement of an infrared focal plane array by use of the self-imaging property of a canted periodic target, Appl. Opt. 38, 631-637 (1999)

7. M. Marchywka, D.G. Socker, Modulation transfer function measurement techniques for small-pixel detectors, Appl. Opt. 31, 7198-7213 (1992)

8. J.E. Greivenkamp, A.E. Lowman, Modulation transfer function measurements of sparse-array sensors using a self-calibrating fringe pattern, Appl. Opt. 33, 5029-5036 (1994)
9. H. Kubota, H. Ohzu, Method of Measurement of Response Function by Means of Random Chart, J. Opt. Soc. Am. 47, 666-667 (1957)

10. G.D. Boreman, E.L. Dereniak, Method for measuring modulation transfer function of charge-coupled devices using laser speckle, Opt. Eng. 25, 148-150 (1986)

11. G.D. Boreman, Y. Sun, A.B. James, Generation of laser speckle with an integrating sphere, Opt. Eng. 29, 339-342 (1990)

12. A.M. Pozo, A. Ferrero, M. Rubiño, J. Campos, A. Pons, Improvements for determining the modulation transfer function of charge-coupled devices by the speckle method, Opt. Express 14, 5928-5936 (2006)

13. A. Daniels, G.D. Boreman, A.D. Ducharme, E. Sapir, Random transparency targets for modulation transfer function measurement in the visible and infrared regions, Opt. Eng. 34, 860-868 (1995)

14. G.D. Boreman, Fourier spectrum techniques for characterization of spatial noise in imaging arrays, Opt. Eng. 26, 985-991 (1987)

15. X. Chen, N. George, G. Agranov, C. Liu, B. Gravelle, Sensor modulation transfer function measurement using band-limited laser speckle, Opt. Express 16, 20047-20059 (2008)

16. A.M. Pozo, M. Rubiño, Comparative analysis of techniques for measuring the modulation transfer functions of chargecoupled devices based on the generation of laser speckle, Appl. Opt. 44, 1543-1547 (2005)

17. M. Sensiper, G.D. Boreman, A.D. Ducharme, D. Snyder, Modulation transfer function testing of detector arrays using narrowband laser speckle, Opt. Eng. 32, 395-400 (1993)

18. J.R. Janesick, Scientific Charge-Coupled Devices, SPIE Optical (Engineering Press, Washington, USA, 2001)

19. W. Astar, New power-efficient optical filter for detector array modulation transfer function measurement by laser speckle, Opt. Eng. 35, 2761-2679 (1996)

20. O. Yadid-Pecht, Geometrical modulation transfer function for different pixel active area shapes, Opt. Eng. 39, 859865 (2000)

21. A.M. Pozo, M. Rubiño, Optical characterization of ophthalmic lenses by means of modulation transfer function determination from a laser speckle pattern, Appl. Opt. 44, 7744-7748 (2005)

22. A.D. Ducharme, Microlens diffusers for efficient laser speckle generation, Opt. Express 15, 14573-14579 (2007)

23. J.W. Goodman, Statical properties of laser speckle and related phenomena, in Laser Speckle and Related Phenomena, Topics in Applied Physics, edited by J.C. Dainty (Springer-Verlag, Berlin, 1984), Vol. 9, pp. 35-40

24. L.I. Goldfischer, Autocorrelation function and power spectral density of laser-produced speckle patterns, J. Opt. Soc. Am. 55, 247-253 (1965)

25. A. Nasibov, A. Kholmatov, H. Nasibov, F. Hacizade, The influence of CCD pixel binning option to its modulation transfer function, Presented at SPIE-2010, Optics, Photonics, and Digital Technologies for Multimedia Applications (Brussels, Belgium 2010), pp. 7723, 77231A$77231 \mathrm{~A}-8$

26. A. Fernández-Oliveras, A.M. Pozo, M. Rubiño, Analysis of the modulation transfer function spectral variation in different detector arrays by means of speckle patterns, J. Imaging Sci. Technol. 53, 031101 (2009) 